\title{
TOLERÂNCIA AO FRIO E CARACTERIZAÇÃO DE HÍBRIDOS ENTRE LEUCAENA LEUCOCEPHALA E L. DIVERSIFOLIA
}

\author{
COLD TOLERANCE AND CHARACTERIZATION OF HYBRIDS \\ BETWEEN LEUCAENA LEUCOCEPHALA AND L. DIVERSIFOLIA
}

\section{Carine Simioni $^{1}$ Nilton Rodrigues Paim ${ }^{2}$ Maria Teresa Schifino-Wittmann ${ }^{3}$}

RESUMO

\begin{abstract}
O objetivo deste experimento foi identificar material promissor para ser utilizado como uma forrageira alternativa nas condições do sul do Brasil. Uma população de híbridos, em gerações avançadas, entre Leucaena leucocephala $e \quad L$. diversifolia (ambas espécies tetraplóides, com 2n=104 cromossomos) foi avaliada quanto à taxa de crescimento em altura, época de florescimento e frutificação, número de flores e de legumes por inflorescência e de sementes por legume, tolerância ao frio (expressa em retenção de folhas durante o inverno) e capacidade de rebrote após o inverno. Foi detectada grande variabilidade intrapopulacional para todas as características avaliadas. Foram identificados indivíduos, reunindo tolerância a frio com características desejáveis, que podem ser utilizados na continuidade dos trabalho de seleção e melhoramento e comprovam a potencialidade de Leucaena como espécie forrageira para o sul do Brasil.
\end{abstract}

Palavras-chave: Leucaena, híbridos, tolerância ao frio, características agronômicas.

\section{SUMMARY}

The objective of this experiment was to identify material to be used as an alternative forage in the conditions of Southern Brazil. A population of hybrids between $\boldsymbol{L}$. leucocephala and L. diversifolia (both tetraploid species, with $2 n=104$ chromosomes) in advanced generations was evaluated. The rate of growth in height, duration of flowering and frutification, number of flowers and legumes per inflorescence, seeds per legume, cold tolerance (expressed as foliage retention during winter) and regrowth ability in early spring were evaluated. A great intrapopulational variability was detected for all evaluated characteristics. Some individuals allying cold tolerance and desirable characteristics were identified to be used for further selection and breeding, which confirms the potentiality of Leucaena as a forage for Southern Brazil.

Key words: Leucaena, hybrids, cold tolerance, agronomic characteristcs.

\section{INTRODUÇÃO}

O Rio Grande do Sul, Estado mais meridional do Brasil, depende basicamente de suas pastagens naturais para a alimentação de bovinos e ovinos. Durante a estação fria, a carência de forragem na pastagem nativa leva à perda de peso e conseqüente diminuição da produção animal. Várias alternativas podem ser empregadas, como suplementação de dieta e manejo adequado das pastagens, entre outras. A possibilidade de utilização de espécies arbóreas, especialmente Leucaena, como um banco de proteína complementar à pastagem nativa, tem despertado o interesse dos pesquisadores. Para tanto é necessária a identificação de materiais tolerantes ao frio.

As espécies do gênero Leucaena Benth (Leguminosae, Mimosoideae) são árvores e arbustos fixadores de nitrogênio de mútiplas utilidades (BREWBAKER, 1989; HUGHES, 1993 e 1998). Além de servirem como forragem e para o enriquecimento do solo, produzem madeira para lenha, combustível, papel, fibra têxtil, assoalhos, postes, móveis e estacas. Podem ser utilizadas como

${ }^{1}$ Bióloga, Mestre em Zootecnia.

${ }^{2}$ Engenheiro Agrônomo, Professor Titular, PhD., Departamento de Plantas Forrageiras e Agrometeorologia, Faculdade de Agronomia, Universidade Federal do Rio Grande do Sul (UFRGS).

${ }^{3}$ Bióloga, Professor Adjunto, Doutor, Departamento de Plantas Forrageiras e Agrometeorologia, Faculdade de Agronomia, UFRGS, CP 776, 91501-970, Porto Alegre, RS, Brasil. E-mail: mtschif@ vortex.ufrgs.br. Autor para correspondência. 
quebra-vento, cerca-viva, para sombra e como ornamentais. As sementes e vagens podem ser empregadas para fabricação de corantes, alimentação humana e artesanato (ALCÂNTARA \& BUFARAH, 1982; MITIDIERI, 1983; NATIONAL ACADEMY OF SCIENCES, 1984; BREWBAKER, 1989). A espécie mais conhecida e largamente cultivada é L. leucocephala, a qual parece ter evoluído sob cultivo (HUGHES \& HARRIS, 1995). Porém, apresenta limitações de utilização em locais frios, com solos ácidos, além da conhecida suscetibilidade ao psilídeo Heteropsylla cubana, relaciondas à sua estreita base genética fora de seu habitat nativo (HELLIN \& HUGHES, 1992; HUGHES, 1993, 1998; SORENSSON, 1995).

Devido à alta qualidade e rendimento de sua forragem, esta espécie comporta um programa de melhoramento, com a finalidade de adaptá-la a locais onde as condições de clima e de solo não sejam as mais adequadas. Hibridações interespecíficas são uma alternativa para o melhoramento vegetal, e em Leucaena parece não haver barreiras genéticas à hibridação (BREWBAKER \& SORENSSON, 1994; SORENSSON, 1995).

Algumas espécies de Leucaena podem suportar geadas freqüentes e regulares, com temperaturas mínimas de até $-15^{\circ}$. L . diversifiolia é uma espécie de locais altos, que cresce em condições frias mas sem geadas (HUGHES,1993). O híbrido KX3, entre $L$. leucocephala e $\boldsymbol{L}$. diversifolia sobreviveu a geadas e temperaturas mínimas de até $-12^{\circ}$ C, nas condições de Queensland, Austrália (GUTTERIDGE \& SORENSSON, 1992).

Híbridos entre $\boldsymbol{L}$. leucocephala e $\boldsymbol{L}$. diversifolia são promissores, pois apresentam boa produtividade e valor nutritivo (características da primeira espécie) aliados a vigor e tolerância ao frio (herdados da segunda espécie), mostrando uma combinação desejável de características, sem perda de fertilidade (HUGHES, 1993, 1998).

No Departamento de Plantas Forrageiras e Agrometeorologia, vem-se trabalhando com Leucaena desde 1982, com diversas abordagens como citogenética, eletroforese de isozimas e caracterização morfológica e agronômica (FREITAS et al., 1988, 1991a/b, 1995; SCHIFINOWITTMANN \& SCHLEGEL, 1990; SCHIFINOWITTMANN et al., 1995a/b, 1996; SIMIONI, 1996). Estão em andamento, também, projetos de utilização de material selecionado em sistemas silvipastoris, em áreas de campo nativo.

FREITAS et al. (1991a/b, 1995) analisando uma população de 225 híbridos (51 genótipos), entre $\boldsymbol{L}$. leucocephala e $\mathbf{L}$. diversifolia (ambas tetraplóides, com $2 \mathrm{n}=104$ ), encontraram alguns indiví- duos que reuniam boa produção de folhagem e tolerância ao frio, manifestada pela pouca perda de folhas durante o inverno e pelo vigoroso rebrote após o mesmo.

O objetivo do presente trabalho foi avaliar gerações avançadas de alguns dos híbridos selecionados por FREITAS et al. (1991 a e b; 1995), para verificar a variabilidade existente em características morfológicas, reprodutivas e agronômicas, e identificar aqueles indivíduos que reunissem tolerância ao frio com características desejáveis para serem utilizados como forrageiras no Rio Grande do Sul.

\section{MATERIAL E MÉTODOS}

Os cruzamentos originais foram realizados pelo Dr. E.M. Hutton no CIAT, Cali, Colômbia, utilizando L. leucocephala como progenitor feminino e $\boldsymbol{L}$. diversifolia como progenitor masculino. Ao amanhecer, inflorescências de $\boldsymbol{L}$. leucocephala em início de antese foram mergulhadas em uma solução detergente gametocida. Os grãos de pólen de $\boldsymbol{L}$. diversifolia foram aplicados liberalmente nas flores emasculadas. As inflorescências com flores polinizadas foram ensacadas por um período de 24 a $48 \mathrm{~h}$ (HUTTON ${ }^{\mathrm{a}}, 1986$ - Informe Verbal).

A população original de FREITAS et al. (1991a/b, 1995) foi estabelecida a partir das sementes enviadas pelo Dr. Hutton e contituía-se de árvores F3, F4 e F5. Para o presente trabalho, plantas jovens obtidas a partir de sementes (progênies) de 20 dessas árvores selecionadas, representando as gerações F4, F5 e F6, e denominadas de genótipos 1.1, $6.5,9.5,19.3,23.1,23.2,24.5,26.3,35.3,46.2,46.4$, $46.5,48.2,49.1,50.1,50.3,50.4,50.5,51.1$ e 51.3 foram dispostas em parcelas. As parcelas continham cinco indivíduos, no delineamento de blocos completos casualizados, com 6 repetições, num total de 600 plantas. O espaçamento entre plantas foi de $1,5 \mathrm{~m}$ entre elas e corredores de circulação de $3 \mathrm{~m}$ entre os blocos.

O experimento foi estabelecido em 28 de dezembro de 1993 na Estação Experimental Agronômica da Universidade Federal do Rio Grande do Sul, no município de Eldorado do Sul, Rio Grande do Sul, a $30^{\circ} 05^{\prime} 22^{\prime \prime}$ de latitude sul , $51^{\circ} 39^{\prime} 08^{\prime \prime}$ de longitude oeste e altitude média de $46 \mathrm{~m}$, e clima do tipo Cfa, sendo junho e julho os meses mais frios e janeiro e fevereiro os mais quentes. O solo da área experimental, do tipo laterita hidromórfico, não sofreu correções nem adubações. Em setembro de 1994, após o inverno, foi realizado um corte de uniformização a $40 \mathrm{~cm}$ de altura.

As características analisadas dividiram-se em quatro grupos: (a) taxas crescimento em altura, 
medidas no período vegetativo (do corte até o início do florescimento) e no período reprodutivo (durante o florescimento e frutificação); (b) época do início e duração do florescimento (compreendido entre o aparecimento dos primeiros botões e abertura das últimas inflorescências) e duração da frutificação (compreendida entre surgimento dos primeiros legumes e maturação dos últimos); (c) determinação do número de flores por inflorescência, legumes por inflorescência e sementes por legume (em amostras de 10 inflorescências e de 10 legumes por planta); (d) retenção de folhas e capacidade de rebrote após o inverno.

Durante o inverno de 1994, meses de junho, julho e agosto, com temperaturas máximas, mínimas e médias de, respectivamente $9,0^{\circ} \mathrm{C}, 17,4^{\circ} \mathrm{C}$ e $12,9^{\circ} \mathrm{C}$ em junho, $8,8^{\circ} \mathrm{C}, 17,4^{\circ} \mathrm{C}$ e $12,8^{\circ} \mathrm{C}$ em julho e $9,6^{\circ} \mathrm{C}, 19,6^{\circ} \mathrm{C}$ e $14,0^{\circ} \mathrm{C}$ em agosto; e inverno de 1995, meses de maio, junho, julho e agosto, com temperaturas máximas, mínimas e médias de, respectivamente $8,5^{\circ} \mathrm{C}, 21,6^{\circ} \mathrm{C}$ e $14,6^{\circ} \mathrm{C}$ em maio, $7,2^{\circ} \mathrm{C}, 19,3^{\circ} \mathrm{C}$ e $12,8^{\circ} \mathrm{C}$ em junho, $9,7^{\circ} \mathrm{C}, 20,8^{\circ} \mathrm{C}$ e $14,6^{\circ} \mathrm{C}$ em julho e $9,2^{\circ} \mathrm{C}, 20,8^{\circ} \mathrm{C}$ e $14,3^{\circ} \mathrm{C}$ em agosto.

As plantas avaliadas através de uma escala visual: 0 , plantas com perda total das folhas; $25 \%, 50 \%, 75 \%$ e $100 \%$ correspondendo a plantas que mantiveram respectivamente, $25 \%, 50 \%, 75 \%$ e $100 \%$ de suas folhas verdes. A capacidade de rebrote também foi estimada por escala visual: 0, ausência de rebrote inicial, $50 \%$ e $100 \%$, plantas que apresentaram 50 e $100 \%$ de rebrote, respectivamente. Verificou-se também o número de semanas necessárias para o rebrote, a partir da última semana de inverno; a última observação foi realizada quando todas as plantas sobreviventes haviam rebrotado, ou seja 10 semanas em 1994, seis semanas em 1995.

Os dados referentes às características quantitativas foram submetidos à análise de variância. Quando o teste F foi significativo, a análise foi complementada pelo teste de agrupamentos de Scott-Knott.

\section{RESULTADOS E DISCUSSÃO}

As médias gerais para as taxas de crescimento em altura foram de 6.24 e $11,52 \mathrm{~cm}$. semana ${ }^{-1}$ nos períodos vegetativo e reprodutivo, respectivamente (figura 1 ). $\mathrm{O}$ teste de agrupamentos de Scott-Knott identificou três grupos de médias para a taxa de crescimento em altura no período vegetativo, sendo que aquele com as maiores taxas médias $(7,73$ e $9,93 \mathrm{~cm}$. semana $^{-1}$ ) incluía os genótipos 1.1, 48.2, 9.5, 50.4, $6.5,23.2$ e 23.1. No período reprodutivo também foram identificados três grupos. Naquele das maiores médias $\left(11,65\right.$ a $15,85 \mathrm{~cm}$. semana $\left.^{-1}\right)$ estavam incluídos, além dos genótipos 1.1, 48.2, 9.5, 50.4, 6.5, 23.2 e 23.1, também os genótipos 46.2, 26.3, 46.5 e 50.1. Para todos os tratamentos, e na média geral, os maiores valores foram observados na fase reprodutiva. Em várias espécies, é neste período que ocorre uma maior mobilização de hormônios de crescimento dos caules (FERRI, 1986), que se alongam com maior velocidade em direção à luz, necessária à formação das flores (STREET \& OPIK, 1974). Fortes intensidades de luz favorecem o crescimento de Leucaena (NATIONAL ACADEMY OF SCIENCES, 1984), e a temperatura ótima situase entre entre $22-30^{\circ} \mathrm{C}$ (SKERMAN, 1977), o que está de acordo com o observado neste trabalho, ou seja maiores taxas de crescimento durante a primavera e verão, que coincide com o período reprodutivo e com maiores temperaturas e fotoperíodos.

Quanto à época de florescimento e frutificação , 94,83\% da população floresceu entre outubro de 1994 e agosto de 1995, aproximadamente um ano após o plantio. O florescimento não foi simultâneo para todas as plantas e progênies, e concentrou-se nos meses de final de primavera e verão (novembro e dezembro de 1994), o que está de acordo com observações de GUPTA \& PATIL, 1984, CHANDRASEKHARAN, GOVINDASWAMY (1985); FREITAS et al. (1991a; 1991b; 1995). A duração do florescimento variou de 134,19 dias (genótipo 23.1) até 238,51 dias (genótipo 24.5), com

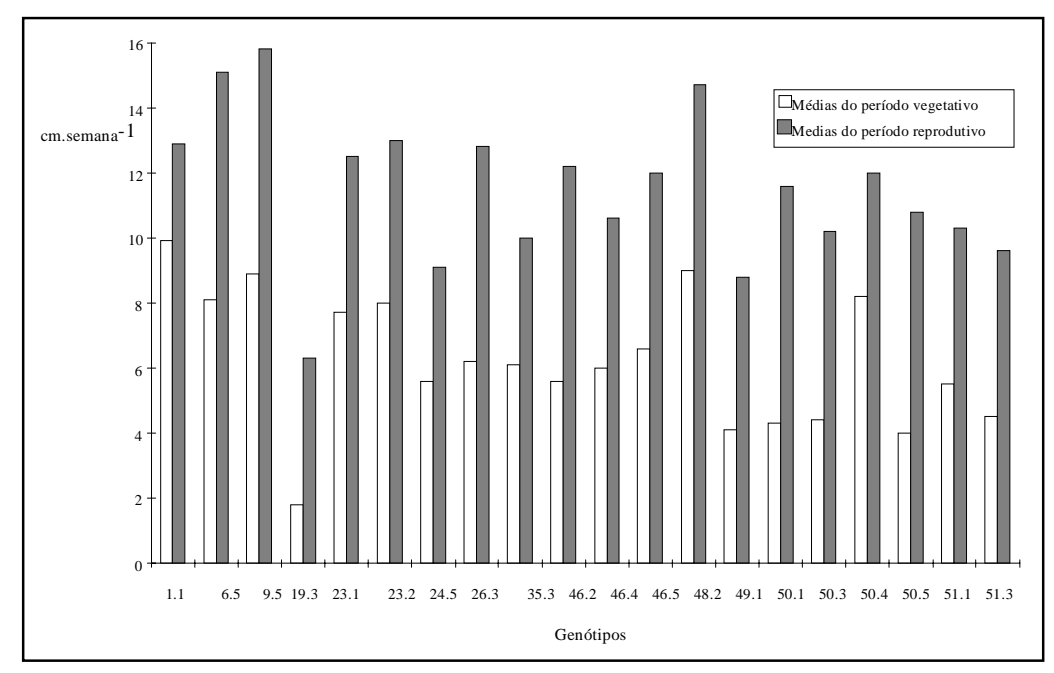

Figura 1 - Taxas de crescimento em altura nos períodos vegetativo e reprodutivo dos indivíduos da população híbrida agrupados pela média de cada tratamento (genótipo). EEA-UFRGS, 1994/1995. 
uma média geral de 183,5 dias, o que certamente reflete diferenças genéticas entre os diversos genótipos. A época da frutificação estendeu-se de novembro de 1994 a agosto de 1995 e 94,74\% das plantas que floresceram no primeiro ano produziram vagens.

$\mathrm{Na}$ população estudada, florescimento e frutificação ocorreram simultaneamente. Em algumas espécies de Leucaena, o florescimento e frutificação são restritos a algumas épocas determinadas e em outras pode haver uma distribuição mais ampla ao longo do ano, com florescimento e frutificação simultâneos (HUGHES,1998). Em termos aplicados, a floração prolongada pode ser favorável para a produção de sementes; por outro lado, um período vegetativo maior pode ser interessante para a produção de forragem.

Na tabela 1, são apresentados os valores para números de flores por inflorescência, de legumes por inflorescência e de sementes por legume. Para número de flores por inflorescência, a média da população foi de $109,43(82,71$ a 134,88$)$. Para número de legumes por inflorescência, a média da população foi de $3,63(1,85$ a 7,06$)$. Nem sempre a maior produção de flores implicou maior produção de frutos e, em geral, apenas 3,28\% das flores pro-

Tabela 1 - Valores médios de número de flores por inflorescência, legumes por inflorescência e sementes por legume na população avaliada de híbridos entre Leucaena leucocephala e $L$. diversifolia.

\begin{tabular}{|c|c|c|c|}
\hline $\begin{array}{l}\text { Genó- } \\
\text { tipo }\end{array}$ & $\begin{array}{l}\mathrm{n}^{\circ} \text { de flores por } \\
\text { inflorescência }\end{array}$ & $\begin{array}{c}\mathrm{n}^{\circ} \text { de legumes } \\
\text { por inflorescên- } \\
\text { cia }\end{array}$ & $\begin{array}{c}\mathrm{n}^{\circ} \text { de sementes } \\
\text { por legume }\end{array}$ \\
\hline 1.1 & $89,88 \mathrm{c}$ & $5,96 a$ & $12,40 \mathrm{~b}$ \\
\hline 6.5 & $110,08 \mathrm{~b}$ & $5,01 \mathrm{a}$ & $13,59 a$ \\
\hline 9.5 & $100,48 \mathrm{~b}$ & $3,73 \mathrm{~b}$ & $13,92 \mathrm{a}$ \\
\hline 19.3 & $117,58 \mathrm{a}$ & $2,70 \mathrm{~b}$ & $14,65 \mathrm{a}$ \\
\hline 23.1 & $109,31 \mathrm{~b}$ & $7,06 a$ & $11,17 \mathrm{~b}$ \\
\hline 23.2 & $94,35 \mathrm{c}$ & $2,45 \mathrm{~b}$ & $14,44 \mathrm{a}$ \\
\hline 24.5 & $82,71 \mathrm{c}$ & $1,85 \mathrm{~b}$ & $13,40 \mathrm{~b}$ \\
\hline 26.3 & $98,30 \mathrm{c}$ & $2,65 \mathrm{~b}$ & $12,66 \mathrm{~b}$ \\
\hline 35.3 & $133,96 \mathrm{a}$ & $2,70 \mathrm{~b}$ & $16,47 \mathrm{a}$ \\
\hline 46.2 & $102,50 \mathrm{~b}$ & $5,78 \mathrm{a}$ & $11,81 \mathrm{~b}$ \\
\hline 46.4 & $124,15 \mathrm{a}$ & $2,96 \mathrm{~b}$ & $16,68 \mathrm{a}$ \\
\hline 46.5 & $103,61 \mathrm{~b}$ & $4,25 \mathrm{~b}$ & $14,19 \mathrm{a}$ \\
\hline 48.2 & $97,13 \mathrm{c}$ & $2,96 \mathrm{~b}$ & $14,24 \mathrm{a}$ \\
\hline 49.1 & $131,13 \mathrm{a}$ & $2,53 \mathrm{~b}$ & $15,55 \mathrm{a}$ \\
\hline 50.1 & $133,06 a$ & $5,00 \mathrm{a}$ & $14,42 \mathrm{a}$ \\
\hline 50.3 & $95,08 \mathrm{c}$ & $2,85 \mathrm{~b}$ & $13,63 \mathrm{~b}$ \\
\hline 50.4 & $95,55 \mathrm{c}$ & $2,86 \mathrm{~b}$ & $15,67 \mathrm{a}$ \\
\hline 50.5 & $103,30 \mathrm{~b}$ & $3,23 \mathrm{~b}$ & $14,11 \mathrm{a}$ \\
\hline 51.1 & $134,88 \mathrm{a}$ & $4,08 \mathrm{~b}$ & $13,78 \mathrm{a}$ \\
\hline 51.3 & $131,53 \mathrm{~b}$ & $1,91 \mathrm{~b}$ & $11,47 \mathrm{~b}$ \\
\hline $\begin{array}{c}\text { média } \\
\text { geral }\end{array}$ & 109,43 & 3,63 & 13,91 \\
\hline
\end{tabular}

Médias na mesma coluna seguidas de mesma letra não diferem significativamente pelo teste de agrupamento de Scott-Knott a $5 \%$ de probabilidade. duziram legumes (considerando-se as médias gerais apresentadas na tabela 1), o que está de acordo com MAMICPIC, EMPIG (1980), que verificaram, em $\boldsymbol{L}$. leucocephala, produção de $3 \%$ de vagens em relação ao número de flores por inflorescência. O número de sementes por legume foi, em média, 13,86 $(11,17$ a 16, 68), mostrando estabilidade em relação ao número observado na população original $(12,62)$, conforme relatado por FREITAS et al. (1991 a ,b e 1995).

Quanto à retenção de folhas e capacidade de rebrote após o inverno, $8,75 \%$ das plantas mantiveram entre 100 e $50 \%$ de suas folhas durante o inverno de 1994 e 6,82 \% durante o inverno de 1995. Em 1994, 95,8\% dos indivíduos apresentaram rebrote total (ou seja, homogêneo ao longo do caule) 10 semanas após a passagem do inverno, sendo que $54,9 \%$ destas plantas necessitaram de apenas duas semanas para rebrotação total. Em 1995, 49,45\% dos indivíduos rebrotaram totalmente em apenas duas semanas após o inverno, atingindo um total de $93 \%$ dos indivíduos seis semanas após o fim do inverno. Apenas 38 plantas $(7,0 \%)$ não rebrotaram.

Com base nas diferenças observadas quanto à capacidade de retenção de folhas e na velocidade de rebrote, verifica-se haver, nessa população, uma grande variabilidade quanto à tolerância ao frio entre as progênies e também entre os indivíduos de cada genótipo. A avaliação da persistência das folhas durante os períodos frios identificou aqueles mais tolerantes. Na tabela 2, são apresentadas algu-

Tabela 2 - Plantas da população híbrida selecionadas por suas características de tolerância ao frio, rebrotação e produção de sementes.

\begin{tabular}{rl}
$\begin{array}{c}\text { Tratamentos } \\
\text { (genótipos) }\end{array}$ & \multicolumn{1}{c}{ Número da planta correspondente } \\
& \\
1.1 & $4,6,10,20^{*}, 26$ \\
6.5 & $3^{*}, 7,10,14,17,19,25$ \\
9.5 & $1,2,9,14,17,21,22,24,25,28,30$ \\
23.1 & 10,17 \\
23.2 & $2,4,5,10,15,16,17,19,23,25,26$ \\
24.5 & $1,4,5,6,7,10,11,14^{*}, 17,20^{*}, 21,22,23,26$ \\
26.3 & 7,18 \\
35.3 & 10,23 \\
46.5 & 2,15 \\
48.2 & $1,3,7,8,9,10,13,15,20,28,30$ \\
49.1 & 14,16 \\
50.3 & $3,5,28,30$ \\
50.4 & $9 *, 14,17,22,23,28$ \\
50.5 & $7,9,16$ \\
51.1 & 5 \\
51.3 & 27
\end{tabular}

* Plantas que permaneceram com 75 ou $100 \%$ de suas folhas durante os invernos de 1994 e 1995. 
mas das plantas que se destacaram na frutificação, mas sem produção excessiva de vagens, com retenção de $25,50,75$ ou até $100 \%$ de suas folhas durante o inverno; rebrote total em duas semanas após o inverno, considerando-se o $2^{\circ}$ ano de avaliações para este critério. Desses indivíduos, os de números 1.1$20,6.5-3,24.5-14$ e 20 e 50.4-9 são os sugeridos para serem utilizados na continuidade do trabalho de seleção e melhoramento, buscando plantas com maior capacidade de retenção de folhas durante o inverno e rebrote após o mesmo.

\section{CONCLUSÃO}

Dentro da população de híbridos entre $\boldsymbol{L}$. leucocephala e L. diversifolia, observam-se indivíduos que apresentaram certa tolerância ao frio (retenção de folhas) durante o inverno. A alta capacidade de rebrote da população, demonstra a potencialidade de utilização de híbridos de $\boldsymbol{L}$. leucocephala e L. diversifolia como material forrageiro alternativo para as condições do Sul do Brasil.

\section{INFORME VERBAL}

a - HUTTONN, E.M. Indooroopilly, Queensland, Austrália, 1986.

\section{REFERÊNCIAS BIBLIOGRÁFICAS}

ALCÂNTARA, P.B., BUFARAH, G. Plantas forrageiras: gramíneas e leguminosas. São Paulo: Nobel, 1982. 150 p.

BREWBAKER, J.L. Leucaena: Can there be such a thing as a perfect tree? Agroforestry Today, Kenya, v. 4, p. 4-7, 1989.

BREWBAKER, J.L., SORENSSON, C.T. Domestication of lesser know species in the genus Leucaena. In: LEAKEY, R., NEWTON, A. (Eds.). Tropical trees: the potencial for domestication. Edinburgh: Institute of Terrestrial Ecology, 1994. p 195-204.

CHANDRASEKHARAN, N.R., GOVINDASWAMY, M Ocurrence of mimosine in the leaves of some spp of Leucaena and hybrids derivates of $\boldsymbol{L}$. diversifolia and $\boldsymbol{L}$. leucocephala. Leucaena Res Rep, v. 6, p. 25-28, 1985.

FERRI, M.G. Fisiologia Vegetal. São Paulo: EPU, 1986. 401 p.

FREITAS, L.H.C., SCHIFINO-WITTMANN, M.T., HUTTON, E.M. Cytogenetic analysis of species and hybrids of Leucaena (Leguminosae) in relation to acid soil tolerance. Rev Bras Genet, v. 11, p. 97-109, 1988.

FREITAS, L.H.C. de, PAIM, N.R., SCHIFINO-WITTMANN, M.T. Morphological characterization and cytogenetical analysis of Leucaena leucocephala $\mathrm{X}$ tetraploid $\boldsymbol{L}$. diversifolia. Leucaena. Res Rep, v. 12, p. 16-18, 1991a.
FREITAS, L.H.C. de, SCHIFINO-WITTMANN, M.T., PAIM, N.R. Floral characteristics, cromossome number and meiotic behavior of hybrids between Leucaena leucocephala $(2 \mathrm{n}=104)$ and tetraploid $\boldsymbol{L}$. diversifolia $(2 \mathrm{n}=104)$ (Leguminosae). Rev Bras Gen, v. 14, p. 781-789, 1991 b.

FREITAS, L.H.C. de, PAIM, N.R. SCHIFINO-WITTMANN, M.T. Caracterização morfológica de híbridos de Leucaena leucocephala e Leucaena diversifolia. Pesq Agrop Bras, v. 30, p. 61-68, 1995.

GUPTA, B.K., PATIL, B.D. Performance of the Leucaena species and hybrids. Leucaena Res Rep, v. 5, p. 27-28, 1984

GUTTERIDGE, R.C., SORENSSON, C.T. Frost tolerance of a Leucaena diversifolia $\mathrm{x}$ L. leucocephala hybrid in Queensland, Australia. Leucaena Res Rep, v. 13, p. 29-31, 1992.

HELLIN, J.J., HUGHES, C.E. Leucaena salvadorensis: conservacion y utilizacion en Centro America. Honduras: COHDEFOR - ODA - ESNACIFOR, 1992. 45 p. Serie Miscelanea de CONSEFORH, 15- 15/92.

HUGHES, C.E. Leucaena Genetic Resources: the OFI seeds collections and a synopsis of species characteristics. Oxford: Oxford Forestry Institute, 1993. 117 p.

HUGHES, C.E. Handbook of Leucaena genetic resources. Oxford: Oxford Forestry Institute, 1998. 254 p.

HUGHES, C.E., HARRIS, S.A. Systematics of Leucaena: recent findings and aplications for breeding and conservation. In: SHELTON, H.M., PIGGIN, C.M., BREWBAKER, J.L. (Eds.). Leucaena: opportunities and limitations. Canberra: ACIAR, 1995. p. 54-65.

MAMICPIC, N.G., EMPIG, L.V. Blooming habits of Ipil-Ipil ( $\boldsymbol{L}$. leucocephala) at College Laguna. Leucaena Newsletter, v. 1, p. $20,1980$.

MITIDIERI, J. Manual de gramíneas e leguminosas para pastos tropicais. São Paulo: Nobel, 1983. 198 p.

NATIONAL ACADEMY OF SCIENCES. Leucaena, promising forage and tree crop for the tropics. 2. ed. Washington: National Academy, 1984. 100 p.

SCHIFINO-WITTMANN, M.T., SCHLEGEL, M. Isozyme patterns of Leucaena leucocephala, L. diversifolia and their htbrids selected for acid soil tolerance. Leucaena Res Rep, v. 11, p. 47-49, 1990.

SCHIFINO-WITTMANN, M.T., PAIM, N.R., FREITAS, L.H., et al. Leucaena in Rio Grande do Sul (Southern Brazil). In: SHELTON, H.M., PIGGIN, C.M., BREWBAKER, J.L. (Eds.). Leucaena: opportunities and limitations. Canberra: ACIAR, 1995a. p. 47-53.

SCHIFINO-WITTMANN, M.T., PAIM, N.R., SIMIONI, C., et al. Germplasm evaluation in Southern Brazil for cold and frost tolerance. Leucnet News, v. 2, p. 15-16, 1995b.

SCHIFINO-WITTMANN, FREITAS, L.H.C., SIMIONI, C. Isoenzymatic characterization of hybrids between Leucaena leucocephala and Leucaena diversifolia ssp. diversifolia grown in Rio Grande do Sul (Southern Brazil). Rev Brasil Genet, v. 19, p. 475-478, 1996. 
SIMIONI, C. Avaliação de tolerância ao frio e caracterização de híbridos entre Leucaena leucocephala e L. diversifolia ssp . diversifolia. Porto Alegre, 108 p. Dissertação - Curso de Pósgraduação em Zootecnia, Universidade Federal do Rio Grande do Sul, 1996.

SKERMAN, P.J. Tropical forage legumes. 2. ed. Roma: FAO, 1977. $609 \mathrm{p}$.
SORENSSON, C.T. Potential for improvement of Leucaena through interespecific hybridization. In: SHELTON, H.M., PIGGIN, C.M., BREWBAKER, J.L. (Eds.). Leucaena: opportunities and limitations. Canberra: ACIAR, 1995. p. $47-53$.

STREET,H. E., OPIK, H. Fisiologia das Angiospermas. São Paulo, Polígono, 1974, 332 p.

Ciência Rural, v. 29, n. 3, 1999. 